\title{
Diversificación económica, siembra de maíz y rendimientos de los productores del estado de Tlaxcala, México
}

\section{Economic diversification, maize sowing and yields for the producers from the State of Tlaxcala, Mexico}

\author{
Miguel Ángel Damián-Huato* \\ Benito Ramírez-Valverde** \\ Agustín Aragón-García* \\ Jesús Francisco López-Olguín*
}

\begin{abstract}
This work studied the relation pluri-activity has with the management and productivity of maize, the use of peasant technologies and of the agriculture-livestock synergy. The results point out that $74 \%$ of the maize producers have diversified their activities, $65 \%$ in primary activities, and $35 \%$ in secondary and tertiary activities. Pluri-activity is affecting the specialization of the producer in the adequate management of maize and its productivity, because the technical discontinuity of maize producers has broadened and we found that there is a rupture in the transition of empirical knowledge and peasant technologies, as well as a deterioration in the use of the agriculture-livestock synergy, essential aspects for the management of maize.
\end{abstract}

Keywords: pluri-activity, maize management, specialization, technologic discontinuity, and peasant technologies.

\section{Resumen}

Este trabajo estudió la relación que tiene la pluriactividad con el manejo y productividad del maíz, el empleo de tecnologías campesinas y de la sinergia agricultura-ganadería. Los resultados señalan que: $74 \%$ de los productores de maíz han diversificado sus actividades, $65 \%$ en actividades primarias y $35 \%$ en secundarias y terciarias. La pluriactividad está afectando la especialización del productor en el manejo adecuado del maíz y su productividad, porque se ha ampliado la discontinuidad técnica de los maiceros y se encontró que existe una ruptura en la transmisión de conocimientos empíricos y tecnologías campesinas, así como un deterioro de la sinergia agricultura-ganadería, aspectos esenciales para el manejo del maíz.

Palabras clave: pluriactividad, manejo del maíz, especialización, discontinuidad técnica y tecnologías campesinas.

* Benemérita Universidad Autónoma de Puebla, México. Correos-e: damianhuato@hotmail. com, aragon@siu.buap.mx, cs002116@siu.buap.mx.

** Colegio de Postgraduados, Campus Puebla, México. Correo-e: bramirez@colpos.mx. 


\section{Introducción}

La aplicación de políticas neoliberales en el campo mexicano ha causado grandes cambios, entre las que destaca que la agricultura ha ido perdiendo su papel de producción de alimentos baratos para la población del país, reduciendo sus funciones a la reproducción de fuerza de trabajo. Al perder como productor el vínculo con el capital, las políticas públicas excluyeron a los campesinos de los planes integrales productivos, y los incorporaron a proyectos asistenciales para apoyar su reproducción como fuerza de trabajo y no como productores (Rubio, 2001). Del Roble (2005) indica que el tamaño del gasto público en México se redujo en cerca de $35 \%$ del PIB total a aproximadamente una cuarta parte del mismo; ello también se reflejó en que el gasto público agropecuario pasó de representar casi 6\% del total a sólo 1.3\% del PIB en 2001. El mismo autor señala que el gasto público agropecuario destinado al fomento productivo de la agricultura pasó de poco más de las cuatro quintas partes del total del gasto agropecuario y rural, que detentaba en el promedio trienal 19851987, a dos terceras partes del promedio trienal 1999-2001, en tanto que el rubro del gasto ejercido en inversión social se incrementó de $16 \%$ del monto promedio del trienio inicial, a $28 \%$ del trienio final.

Simultáneamente, las políticas neoliberales han alentado el desarrollo de productores que tienen posibilidades de articularse a los circuitos agroindustriales y agrocomerciales desdeñando el desarrollo de la agricultura de subsistencia productora de granos básicos, lo que ha ocasionado que la población rural mexicana sea cada vez menos agropecuaria. Todavía en 1970 se podía considerar que la población rural trabajaba primordialmente en el campo, ya que $78 \%$ de su población económicamente activa (PEA) laboraba en el sector primario, $9 \%$ en el secundario y $9 \%$ en el sector terciario. En la actualidad la situación ha cambiado totalmente, ya que cerca de la mitad de la PEA en el campo trabaja en los sectores secundario y terciario; llama todavía más la atención la poca importancia del sector agropecuario en las localidades rurales del centro de la República Mexicana (De Grammont, 2009).

Estos cambios han convertido al campo mexicano en reservorio de fuerza laboral cuya reproducción social descansa en la pluriactividad económica. En el capitalismo la diversificación económica siempre ha sido una estrategia fundamental de reproducción social, debido a que los medios de producción son escasos para absorber la totalidad de la fuerza de trabajo y los ingresos agrícolas resultan insuficientes para garantizar la sobrevivencia de los miembros de las familias rurales (Bartra, 2006); no obstante, este proceso se ha acentuado en la fase neoliberal. 
La pluriactividad se define como la ejecución de actividades agropecuarias y no agropecuarias por parte de los productores para garantizar la sobrevivencia de la familia rural (De Grammont, 2009). Es lo que Galindo (1994) denomina agricultura de tiempo parcial, que surge cuando la actividad agrícola se complementa con un trabajo asalariado externo. Gras (2004) la concibe como la combinación de ocupaciones y actividades laborales que desarrollan los productores y sus familias, sea dentro o fuera del predio o dentro o fuera del sector agropecuario. Para Sacco y Velleda (2007), la pluriactividad se encuentra estructuralmente asociada a la agricultura familiar y forma parte de las estrategias de supervivencia adoptadas por los pequeños agricultores y campesinos para garantizar la reproducción social de sus familias. Tales estrategias se han desarrollado mediante la combinación de diversas actividades (agrícolas y no agrícolas) realizadas dentro o fuera de las propias explotaciones. En síntesis, la pluriactividad comprende la ejecución simultánea de diversas actividades económicas, que el productor puede efectuar en su comunidad de origen o fuera de ella, por razón de la migración temporal.

Es cierto que la pluriactividad puede resultar una estrategia eficaz de sobrevivencia para mejorar el ingreso de los hogares rurales; así lo demuestran diversos autores que han estudiado este fenómeno (Vernimmen et al., 2002; De Grammont, 2009; De Janvry y Sadoulet, 2004). Sin embargo, otros estudios (Anseeuw y Laurent, 2007; Deininger y Olinto, 2004) señalan que los hogares que han diversificado sus ingresos, redujeron la renta agrícola y su dependencia de la agricultura es más baja que la peor de los hogares subordinados a las actividades agrícolas. Esto sugiere que la pluriactividad puede ocasionar que el productor, al abandonar (temporal o definitivamente) o descuidar la siembra de plantas, reduzca su especialización en el manejo de los cultivos.

El manejo de los cultivos comprende todas las prácticas agrícolas (preparación del suelo, fecha de siembra, labores de cultivo, fertilización, densidad de siembra, combate de plagas y enfermedades, etc.) que el productor efectúa durante el ciclo agrícola y la forma como combina los factores de la producción en cada una de las actividades ejecutadas. Considerando los factores limitantes de la tierra, el manejo es, en última instancia, el aspecto que determina la producción (Ponce, 1998). Un productor especializado en el manejo de los cultivos se encuentra mejor facultado para innovar, generar o adecuar conocimientos y tecnologías; combinar los factores productivos de forma más eficiente; crear múltiples sinergias que potencian la productividad de los recursos empleados en las actividades agropecuarias, y para acrecentar los ingresos de las familias rurales. 
En México coexisten distintos tipos de manejo de cultivos; en maíz destacan dos que son contrastantes: el convencional o comercial y el campesino o agroecológico.

El primero se caracteriza porque la tecnología utilizada responde a las condiciones de la agricultura comercial fundada en el uso de agroquímicos. Tiene su origen en la Revolución Verde, sostenida mediante el empleo de híbridos que sólo pueden lograr cosechas superiores al de los materiales criollos si disponen de agua y fertilizantes que, a su vez, promueven el crecimiento de malezas y patógenos, cuyo combate exige aplicar herbicidas e insecticidas sintéticos (Borlaug y Dowswell, 2005). En México, este tipo de tecnología la genera y recomienda el Instituto Nacional de Investigaciones Forestal, Agrícola y Pecuaria (INIFAP).

Es cierto que la Revolución Verde mejoró la productividad en el corto y mediano plazos; sin embargo, también ha tenido una secuela de efectos nocivos como: reducción de la productividad en el largo plazo, aumento de los costos de producción, exclusión de productores de las actividades primarias e incremento de las desigualdades sociales en el campo (Rosset, 1997); degradación y salinización de suelos agrícolas, contaminación con fertilizantes y pesticidas, disminución de la biodiversidad, agotamiento y sobreexplotación de acuíferos (Pengue, 2005), así como su importante contribución al cambio climático (Stern, 2007).

El manejo campesino es resultado de sistemas de cognición y prácticas donde se seleccionan las tecnologías más útiles y adaptables a los agroecosistemas. Se trata de innovaciones agroecológicas que: a) ensamblan distintos componentes del agroecosistema (cultivos, suelos, plantas, animales); b) crean sinergias que potencian la productividad de los recursos cuyo costo tiende a cero (energía solar, aire, nitrógeno, carbono, cadenas y tramas tróficas) ejecutando prácticas como asociación y rotación de cultivos, manejo integrado de plagas y enfermedades, uso de estiércol como abono y conservación de suelos y aguas, y c) promueven la relación agricultura-ganadería y la captación de carbono.

En este trabajo se estudió la pluriactividad de los maiceros del estado de Tlaxcala y su efecto en el manejo del maíz, la especialización y productividad, así como en el uso de tecnologías campesinas y de la sinergia agricultura-ganadería. La productividad se estimó mediante los rendimientos por hectárea, ya que es una variable compleja que resume el desempeño de otras como clima, suelos, acceso al capital, programas públicos de fomento agrícola, etc. (Turrent et al., 1999). La mayoría de los datos usados en este estudio se obtuvieron de una encuesta aplicada en 2002 a 1,884 productores de maíz distribuidos en los 60 municipios del estado de Tlaxcala. 


\section{Materiales y métodos}

\subsection{Marco geográfico del estudio}

La investigación se realizó en los 60 municipios del estado de Tlaxcala, que se encuentra situado entre los $97^{\circ} 37^{\prime} 07^{\prime \prime}$ y los $98^{\circ} 42^{\prime} 51^{\prime \prime}$ de longitud oeste, y los $19^{\circ} 05^{\prime} 43^{\prime \prime}$ y los $19^{\circ} 44^{\prime} 07^{\prime \prime}$ de latitud norte y cuenta con tres distritos de desarrollo rural (mapa I).

Limita al sur, este y norte con el estado de Puebla; al noroeste con Hidalgo, y al poniente con el Estado de México. Tlaxcala se encuentra entre los 2,200 y 4,400 metros sobre el nivel del mar, posee un clima templado-húmedo y una precipitación media anual de 711 milímetros; los suelos predominantes son los cambisoles y feozems que cubren, respectivamente, 75 y $22 \%$ del territorio, con una población de 1'068,207 personas, $78 \%$ considerada urbana y $22 \%$ rural (INEGI-GET, 2007). Tiene una extensión territorial de $4,060 \mathrm{~km}^{2}$ que constituye $0.2 \%$ del territorio nacional y una densidad de población de 263 habitantes por kilómetro cuadrado.

El estado de Tlaxcala, ubicado en el centro de México, se caracteriza por la importancia histórica que han tenido sus sectores secundario y terciario en la economía. La creación de riqueza en el estado depende de dichos sectores, ya que entre ambos absorben $81.8 \%$ de la población ocupada y generan $93.4 \%$ del producto interno bruto estatal. El sector primario absorbe $18.2 \%$ de la población ocupada y crea $6.6 \%$ de la riqueza (INEGI-GET, 2007). Por este motivo la población rural tiene mayores posibilidades de conseguir un empleo extrapredio.

Por otra parte, datos del siap (2007) indican que en 2006 el área agrícola del estado era de 243,623 hectáreas, $85 \%$ de temporal y $15 \%$ de riego, donde se sembraron 48 cultivos, entre los que destaca el maíz, ya que entre 1990 y 2003 en promedio se sembraron y cosecharon, respectivamente, 136,513 y 129,506 hectáreas (Sagarpa, 2004).

En el ámbito regional, la entidad se divide en tres distritos de desarrollo rural (DDR): a) el 163, ubicado en Calpulalpan, consta de 11 municipios, concentra $42.3 \%$ del área cosechada y genera $42.8 \%$ del valor de la producción agrícola total del estado; $b$ ) el 164, con sede en la ciudad de Tlaxcala, abarca 36 municipios que aportan $26.2 \%$ del valor de la producción, yc) el 165, que se localiza en Huamantla, incluye 13 municipios y su estructura agrícola se compone de cerca de 29 cultivos que ocupan $30.3 \%$ de la superficie que se cultiva en la entidad. Los DDR son la base territorial para diseñar y operar el Programa Especial Concurrente y los programas sectoriales que de él se derivan del gobierno mexicano. 


\section{Mapa I}

Ubicación del estado de Tlaxcala y los distritos de desarrollo rural

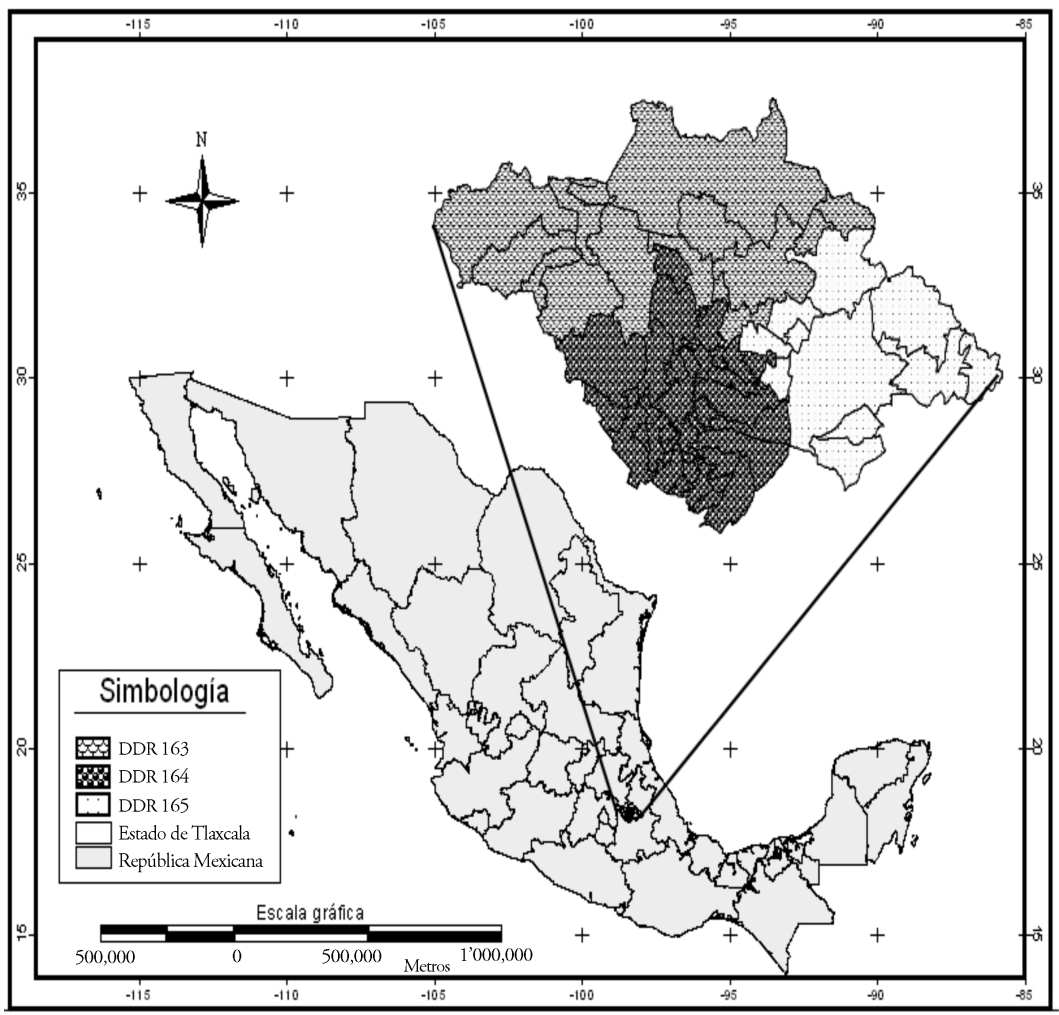

Fuente: Elaborado por Kenia Cuatecontzi Morales, con datos del Economic and Social Research Institute (ESRI), http://www.esri.ie/, 2002.

\subsection{La encuesta}

Ésta consistió en un cuestionario mediante el cual se recopiló y sistematizó la mayor parte de los datos analizados en la investigación. A cada productor de una muestra representativa se aplicó el cuestionario con 125 preguntas de tipo cerrado, el cual se probó antes de su aplicación definitiva efectuada en agosto de 2002.

\subsection{Tamaño de la muestra}

La encuesta se aplicó a una muestra representativa estimada mediante muestreo simple aleatorio, con distribución proporcional al número de productores por municipio y localidad. El marco de muestreo fueron los 94,963 productores de maíz registrados en el Programa Directo de Apo- 
yo al Campo (Procampo) del estado de Tlaxcala. La selección de las unidades de muestreo (productores) se realizó al azar una a una y sin reemplazo. Para evitar que un productor apareciera más de una vez en la muestra, se depuró la lista original que aportó la oficina de Apoyos y Servicios a la Comercialización Agropecuaria. El marco de muestreo resultante fue de 43,274 agricultores.

Para determinar el tamaño de muestra se utilizó la siguiente expresión matemática (Gómez, 1977):

$$
\mathrm{n}=\frac{\mathrm{N} \mathrm{Z}_{\alpha / 2}^{2} \mathrm{~S}_{\mathrm{n}}{ }^{2}}{\mathrm{Nd}^{2}+\mathrm{Z}^{2}{ }_{\alpha / 2} \mathrm{~S}^{2}}
$$

Donde $n$ es el tamaño de la muestra; $N$ es el tamaño de la población (43,274 productores); $d$ es la precisión: $20 \mathrm{~kg} ; Z_{\alpha / 2}$ es el valor de $Z$ en la tabla de la distribución normal estándar para una confiabilidad de $95 \%$, y $S_{n}{ }^{2}=438.44$ estimada con datos de un muestreo preliminar.

El tamaño de la muestra calculada fue de 1,770 productores, la cual se amplió a 1,884 por la posible cancelación de algunas entrevistas y para eliminar los cuestionarios inconsistentes.

\subsection{Indice de apropiación de tecnología agrícola (IATA)}

Con la información recopilada mediante la encuesta se cuantificó el IATA para conocer qué tan adecuadamente los productores manejaron el paquete tecnológico generado y recomendado por el INIFAP. El cálculo del IATA se realizó con el siguiente procedimiento: a) se contrastaron las recomendaciones del INIFAP, para cada una de las actividades del cultivo del maíz, con las que aplica el productor; $b$ ) se asignó un valor nominal al paquete tecnológico de 100 unidades y se ponderó con base en el efecto de cada componente en la productividad del maíz: 10 para fecha de siembra, 20 para variedad, 5 para distancia entre surcos, 5 para distancia entre matas, 5 para número de plantas por mata, 25 y 5 para dosis de fertilización y fecha de aplicación del fertilizante, 6 y 4 para tipo y dosis de herbicida, 6 y 4 para tipo y dosis de insecticida y 5 para combate de enfermedades; c) cada uno de los valores ponderados se dividió entre dos: el primer cociente correspondió al uso de la recomendación y el segundo a su manejo adecuado. Por ejemplo, si un productor empleó el híbrido recomendado por el INIFAP se le asignaron 20 unidades; si utilizó otro híbrido mejorado no considerado en el paquete tecnológico, 10 unidades, y si no empleó material híbrido se calificó con cero. Con lo anterior, el valor del IATA varió entre cero y 100 unidades. 
Para calcular el IATA se utilizó la expresión matemática elaborada por Damián et al. (2004):

$$
\text { IATA }=\left[\sum_{\mathrm{i}=1}^{\mathrm{k}}\left(\mathrm{p}_{\mathrm{i}}\right)\left(\mathrm{SPA}_{\mathrm{i}} / \mathrm{PTA}_{\mathrm{i}}\right)\right.
$$

Donde IATA es el índice de apropiación de tecnología agrícola; $k$ es el número de componentes del paquete tecnológico recomendado por el INIFAP; $\mathrm{p}_{\mathrm{i}}$ es la ponderación otorgada al i-ésimo componente de recomendación; donde:

$\sum_{\mathrm{i}=1}^{\mathrm{k}} \mathrm{pi}=100 ; \mathrm{i}=1,2, \ldots \mathrm{k}$ SPAi es el sistema productivo agrícola para el i-ésimo componente de recomendación; $\mathrm{i}=1,2, \ldots \mathrm{k}$; PTA $_{\mathrm{i}}$ es el paquete tecnológico agrícola para el i-ésimo componente de recomendación; i = $1,2, \ldots \mathrm{k} ;\left(\mathrm{PTA}_{\mathrm{i}} / \mathrm{PTA}_{\mathrm{i}}\right)$ es la proporción de tecnología empleada, que puede tomar valores de cero, para la no apropiación de la tecnología recomendada por el INIFAP; uno, para el uso adecuado de la tecnología y 0.5 para el empleo inadecuado del componente tecnológico.

\subsection{Tipología de productores}

Se usaron dos criterios para agrupar a los maiceros. En el primero se clasificaron según su pluriactividad en: primarios ( $\mathrm{P}$ ) y primarios con otras actividades (POA). En los primeros se incluyen los productores que solamente siembran maíz, así como los que cultivan maíz y realizaron otras tareas de las ramas económicas del sector primario; los POA son quienes ejecutaron otras actividades de los sectores secundario y terciario, además de las dedicadas al cultivo de maíz. El segundo criterio que se empleó fue la ubicación territorial, agrupando a los productores por DDR.

\section{Resultados y discusión}

\subsection{Pluriactividad de los productores de maiz}

Datos de la encuesta muestran que los maiceros del estado de Tlaxcala son pluriactivos, ya que 1,392 (74\%) efectuaron varias actividades, de las cuales seis son las más significativas: jornalero, ganadero, comerciante, albañil, servidor público y recolector de leña, respectivamente desempenadas por el $20,15,11,10,5$ y $3 \%$; otro conjunto de actividades lo realizó otro $10 \%$ de los productores, mientras que el restante 26\% (492), sólo se dedicó a la siembra de maíz. 
Como se dijo, la pluriactividad comprende la ejecución simultánea de diversas actividades económicas que el productor puede efectuar en su comunidad de origen o fuera de ella, por razón de la migración temporal.

\subsection{Pluriactividad en la comunidad de origen del productor}

En el cuadro 1 se muestran los resultados obtenidos cuando los productores son agrupados por el tipo de actividad que efectúan y el distrito de desarrollo rural donde se ubican.

\section{Cuadro 1}

\section{Productores de maíz del estado de Tlaxcala agrupados de acuerdo con las actividades que desempeńan y por DDR}

\begin{tabular}{lcrcrcrcr}
\hline \multirow{2}{*}{ Tipo de productores } & \multicolumn{2}{c}{ Calpulalpan } & \multicolumn{2}{c}{ Tlaxcala } & \multicolumn{3}{c}{ Huamantla } & \multicolumn{2}{c}{ Estado } \\
& Total & $\%$ & Total & $\%$ & Total & $\%$ & Total & $\%$ \\
\hline Primarios & 317 & 68 & 389 & 59 & 512 & 67 & 1,218 & 65 \\
No primarios & 146 & 32 & 266 & 41 & 254 & 33 & 666 & 35 \\
Total & 463 & 100 & 655 & 100 & 766 & 100 & 1,884 & 100 \\
\hline
\end{tabular}

Fuente: Elaboración propia con datos obtenidos de la encuesta.

En estas cifras se puede observar que:

1. El 65\% de los productores realiza actividades exclusivamente relacionadas con el sector primario y $35 \%$ efectúan tareas en diferentes ramas económicas de los sectores secundario y terciario.

2. Los productores P predominan en todos los DDR, y su número es relativamente mayor en los distritos de Calpulalpan y Huamantla. Esta estructura ocupacional presenta ciertas coincidencias con la del INEGi (2001), ya que del total de la población ocupada en el DDR de Calpulalpan, 20, 36 y 42\% efectuaron actividades primarias, secundarias y terciarias, respectivamente, mientras que el resto $(2 \%)$ realizó labores no especificadas. Estos datos para los DDR de Tlaxcala y Huamantla son, en el mismo orden, 20, 36, 42 y $2 \%$; y $38,30,30$ y 2 por ciento.

3. Analizando la muestra de productores estudiada hallamos que, de acuerdo con la prueba de $j i$ cuadrada, hay diferencia estadísticamente significativa $\left(\chi^{2}=12.49 ; \mathrm{p}=0.0019\right)$ en la proporción de maiceros P y POA entre DDR, pero es en el de Tlaxcala donde la pluriactividad se presenta con mayor intensidad, debido a que en éste se localizan los complejos industriales de Panzacola y Xiloxoxtla, Tlaxcala y San Martín Texmelucan, Puebla, construidos du- 
rante la etapa de sustitución de importaciones, ofreciendo mayores posibilidades de empleo a los maiceros.

En el cuadro 2 se exponen los rendimientos por hectárea y el IATA obtenidos por los productores P y POA ubicados por DDR.

Las cifras muestran que, en el ámbito territorial, los maiceros de los DDR de Tlaxcala y Huamantla poseen ligeramente mayor y menor productividad, respectivamente, aun cuando los de Huamantla emplearon más tecnología que los de Tlaxcala. Este hecho se explica más por los rasgos edafoclimáticos de estos DDR, que por la pluriactividad de los productores. Las características edafoclimáticas se pueden estimar mediante el índice de no siniestro (INS), que es el área cosechada como fracción de la superficie cultivada (Turrent et al., 1999). Este índice muestra la incidencia que tienen el suelo y el clima en la productividad agrícola. El cálculo del ins para un promedio de cinco años (INEGI-GET, 1999-2003) seńala que los distritos 165 y 164 poseen los valores extremos: 0.835 el primero y 0.967 el segundo, en tanto que este dato para el DDR 163 fue de 0.908 . Esto significa que el distrito que cultiva maíz en condiciones más adversas es Huamantla, seguido de Calpulalpan y Tlaxcala, ya que 16.5, 9.2 y 3.3\% del área sembrada con maíz en esos DDR no fue cosechada. Estos resultados coinciden con la clasificación que hizo el INIFAP (1999) de los municipios que integran los DDR de la entidad, según su potencial productivo. Una forma de mantener o elevar los rendimientos en regiones que poseen condiciones edafoclimáticas restrictivas es mediante el empleo de más insumos agrícolas, sobre todo fertilizante. Esto explica porqué los productores del DDR 165 poseen mayor IATA, pero menor productividad.

En cuanto a los productores, los datos del cuadro 2 indican que en promedio los maiceros POA obtuvieron significativamente menores rendimientos por hectárea que los $\mathrm{P}$, excepto en el DDR de Calpulalpan, aun cuando los POA emplearon más tecnología convencional. Con respecto al IATA, en ningún DDR se obtuvo diferencia estadística significativa entre los P y POA ( $p>0-05$ en todos los casos). Como se mencionó, el rendimiento es resultado de múltiples factores, entre los que destaca el componente tecnológico. Sin embargo, para que la tecnología influya en los rendimientos se tiene que utilizar de forma adecuada, lo que no ocurrió con los maiceros del estado de Tlaxcala, por lo que la correlación entre IATA y rendimiento es muy débil $(\mathrm{r}=0.135 ; \mathrm{p}<0.001)$.

Por otra parte, resalta que los maiceros POA tienen ciertos atributos (menor edad, mayor escolaridad y gasto), que debería reflejarse en mayores rendimientos unitarios (cuadro 3). Para Rogers y Svenning (1979), estas variables influyen decisivamente en la difusión de innovaciones y en el aumento de la productividad de los agricultores. 

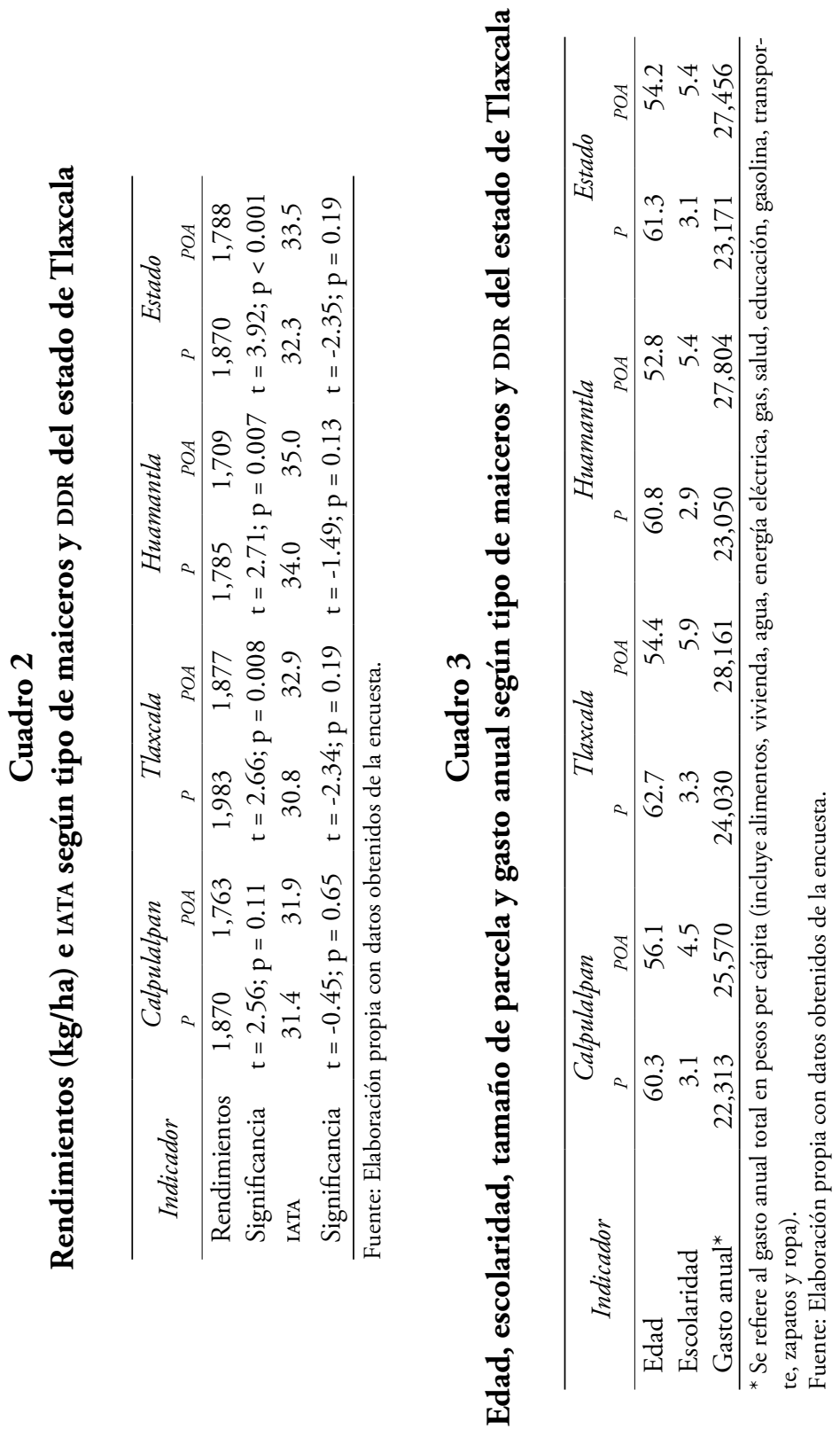
De este modo, los maiceros POA tienen un comportamiento atípico, ya que una mayor disponibilidad de tecnología, así como más educación y gasto debería expresarse en una productividad más alta. Es probable, sin embargo, que el hecho de ejecutar otras actividades distintas a la siembra de maíz afecte la productividad del suelo y, sobre todo, la especialización del productor en el manejo del maíz.

\subsection{Pluriactividad y migración}

La pluriactividad también se puede efectuar fuera de la comunidad de origen del productor, mediante la migración de éste o de algún miembro de la familia, lo que afectará asimismo su productividad.

La emigración de los maiceros en Tlaxcala ha sido promovida por la conjugación de distintos motivos:

a) Los productores poseen pequeñas parcelas agrícolas, 2.34 hectáreas en promedio, pero es un poco mayor $(2.43 \mathrm{ha})$ para los productores que no emigraron y un poco menor $(2.27 \mathrm{ha})$ para los maiceros que sí lo hicieron.

b) Las parcelas que siembran se caracterizan por su baja fertilidad. Ojeda y Ojeda (1996) señalan que los suelos agrícolas del estado son los únicos del país clasificados como de muy baja fertilidad. A esto hay que agregar el grado de erosión que presentan los suelos de Tlaxcala. Datos de la Semarnat (2002) señalan que 392,552 hectáreas, 97\% del área del estado, presentan algún tipo de erosión: $15 \%$ ligera, $74 \%$ moderada y $11 \%$ se considera como severa.

c) La alta densidad de población del estado, que representa la cuarta posición en el país (INEGI-GET, 2001).

d) La entidad se encuentra cerca de los principales polos industriales del país.

e) El estado cuenta con una amplia red carretera y ferroviaria, que ha facilitado su conexión con las principales zonas fabriles del país. Según el Copladet (2004), Tlaxcala tiene un promedio de $60.8 \mathrm{~km}$ de vías de comunicación por cada $100 \mathrm{~km}$ cuadrados.

Datos de la encuesta indican que $17 \%$ de los familiares de los maiceros emigraron y que las remesas que enviaron son básicas para la sobrevivencia familiar, ya que cubren $20 \%$ del gasto total anual per cápita, que es de 4,941 pesos. Pero también es muy probable que las remesas se destinen a la adquisición de insumos agrícolas, como se infiere de los datos expuestos en el cuadro 4, ya que en la medida en que aquéllas aumentan, también crece el uso del componente tecnológico. 


\section{Cuadro 4}

Rendimiento ( $\mathrm{kg} \mathrm{ha}^{1}$ ), IATA y remesas por tipo de productores del estado de Tlaxcala, según el número de emigrantes

\begin{tabular}{lccrrrrrrr}
\hline $\begin{array}{l}\text { Número de } \\
\text { emigrantes }\end{array}$ & \multicolumn{3}{c}{$P$} & & \multicolumn{3}{c}{ POA } & \multicolumn{4}{c}{ Promedio estado } \\
\hline 0 & 1,949 & 31.7 & 0 & 1,753 & 32.5 & 0 & 1,838 & 32.1 & 0 \\
1 & 1,909 & 31.8 & 526 & 1,796 & 34.3 & 669 & 1,828 & 33.2 & 646 \\
2 & 1,922 & 30.4 & 777 & 1,828 & 33.6 & 918 & 1,853 & 32.6 & 879 \\
3 & 1,929 & 34.7 & 1,012 & 1,863 & 34.7 & 963 & 1,902 & 34.6 & 967 \\
Más de 4 & 2,104 & 35.8 & 1,467 & 1,850 & 35.1 & 1,350 & 1,992 & 36.6 & 1,384 \\
\hline
\end{tabular}

Fuente: Elaboración propia con datos obtenidos de la encuesta.

En estos datos se observa que los productores P que no cuentan con emigrantes resultaron, grosso modo, ser los más eficientes en el manejo del maíz, empleando menos tecnología.

\subsection{Pluriactividad, especialización, manejo del maiz y discontinuidad tecnológica}

La pluriactividad afecta la fertilidad del suelo y la especialización de los productores en el manejo del maíz, que en su mayoría son de subsistencia ya que $98 \%$ del grano cosechado se destina al autoconsumo. El primer caso se debe a que cuando los maiceros obtienen de otras actividades la mayoría de sus ingresos, suele suceder que renten o presten su parcela a un familiar o a otro productor, quienes no se preocuparán por mantener o incrementar la fertilidad de un predio que no les pertenece.

Asimismo, el desempeño de otras actividades ocasiona discontinuidad técnica entre los maiceros, la cual se expresa en la paulatina pérdida de su especialización en la siembra de esta gramínea, debido al poco tiempo e interés de que disponen para innovar y mejorar su proceso productivo. Para Smith (1982), el progreso más importante en las facultades productivas del trabajo, y gran parte de la aptitud, destreza y sensatez con que éste se aplica, parecen ser consecuencia de la división del trabajo.

La emigración, a diferencia de la pluriactividad efectuada en la comunidad, ocasiona la ruptura técnica total en el manejo del maíz. Al respecto, Nadal y Wise (2005) plantean que la prolongada interrupción de la siembra de maíz por miembros de la familia, probablemente dificulte la transmisión del conocimiento tradicional, debilitando con ello el manejo apropiado del cultivo.

Una mayor especialización en el manejo del maíz de subsistencia tiene bases distintas a la que impulsaron Taylor y Ford a principios del siglo pasado en el sector secundario, basada en la segmentación de las 
fases del proceso productivo. La línea de montaje y la organización del trabajo se estructuraron a partir del ritmo y requerimientos de la maquinaria utilizada en la industria, mediante la especialización de las funciones, la fragmentación de tareas y la medición de tiempos y movimientos para aumentar la productividad y competitividad de las empresas (Martínez y Montesinos, 1994).

Por el contrario, un productor especializado en el manejo del maíz implica que conoce, empíricamente, las interacciones y sinergias que se establecen entre las distintas prácticas que comprenden el manejo del cultivo y la convergencia de tareas de ramas económicas diversas, como la agricultura y la ganadería. Por ejemplo, un campesino especializado sabe que un barbecho realizado en la fecha adecuada mejora la retención de agua, la aireación y fertilidad del suelo, así como el control natural de plagas y enfermedades.

Es importante subrayar que la disminución del gasto público destinado al campo también ha afectado a los programas de transferencia de tecnología y, probablemente, haya incidido en el aumento de la discontinuidad técnica y en el manejo inadecuado del maíz. En Tlaxcala sólo 158 maiceros tuvieron acceso a la asistencia técnica, de los cuales $79 \%$ son $\mathrm{P}$ y el resto POA.

La pérdida de especialización de los maiceros de Tlaxcala se manifiesta de cinco maneras:

1. En el uso reducido de la tecnología generada y recomendada por el INIFAP. En general, el IATA en el estado de Tlaxcala fue bajo, ya que solamente 32.7 unidades del paquete tecnológico fue utilizado por los maiceros.

2. En la amplia variación observada en las prácticas agrícolas que utilizan en el manejo del maíz: cinco fechas de siembra, 13 híbridos, 17 y 20 distancias entre surcos y matas, cuatro cantidades de plantas por mata, siete tipos y 10 dosis de herbicidas, 58 fórmulas y 12 fechas de aplicación de fertilizante y 12 tipos y 18 dosis de insecticidas.

3. En el manejo inadecuado de la tecnología convencional recomendada por el INIFAP, ya que sólo en la fecha de siembra la usaron correctamente 1,751 (92.9\%) maiceros, mientras que este dato fue de $3.8 \%$ para las variedades mejoradas, $33.7 \%$ para la densidad de plantas, $0 \%$ para la fórmula de fertilizante, $21.5 \%$ para la fecha de aplicación de los fertilizantes, $0.5 \%$ para tipo de insecticida y $0.3 \%$ para la dosis de insecticida. El empleo inadecuado del paquete tecnológico ha ocasionado un uso dilapidador de agroquímicos y mayores daños a los recursos naturales, la diversidad ecológica, la calidad del aire y la sostenibilidad de la agricultura. 
4. En la disminución del uso de tecnologías campesinas en el manejo del maíz.

5. Y en la ruptura de la sinergia agricultura-ganadería.

\subsection{Pluriactividad, tecnologías campesinas, manejo del maiz y productividad}

La pluriactividad también está afectando el empleo de tecnologías campesinas, las cuales son esenciales para mejorar el manejo del maíz y la productividad. Estas tecnologías han sido generadas por millones de campesinos que habitan en pequeñas comunidades rurales que durante milenios las han mejorado, mediante la generación y transferencia del conocimiento empírico.

Entre las tecnologías campesinas empleadas en Tlaxcala destacan la semilla criolla, la asociación y rotación de cultivos, las técnicas de conservación de suelo y la aplicación de estiércol utilizadas, respectivamente, por 92, 65, 76, 63 y 66\% de los maiceros (cuadro 5). Para conocer el empleo de estas tecnologías por los productores sólo se calculó el porcentaje que usó la práctica correspondiente. Para el caso de aspectos cuantitativos, se clasificó como que usa la práctica si aplicó el producto independientemente de la cantidad.

En estos datos se nota que un menor porcentaje de maiceros POA, respecto de los P, aplican tecnologías campesinas, excepto semilla criolla, y es más notorio en el uso de estiércol, debido a que los primeros poseen menos número de cabezas de ganado mayor (cuadro 6). No obstante, lo que sobresale en los datos del cuadro 5 es que el uso de estas tecnologías resultaron más eficientes, ya que todos los rendimientos promedio de los productores P se encuentran por arriba de la media estatal $\left(1,841 \mathrm{~kg} \mathrm{ha}^{1}\right)$ y de la de los maiceros POA.

El predominio actual de las tecnologías campesinas en el manejo del maíz se explica porque se inserta en una cosmovisión rural fundada en una triple relación: sociedad-naturaleza-sistema productivo, de donde se han originado saberes, tecnologías, mitos, rituales y formas particulares de convivencia social. Sin embargo, su persistencia se debe a que promueven distintas interacciones que mejoran el manejo del maíz y su productividad.

Los cultivos asociados maximizan las interacciones agronómicas ya que el agrupamiento de plantas con diferentes hábitos de crecimiento, follajes y estructuras radiculares emplea de forma más eficiente los nutrientes, agua y energía solar (Altieri, 1991). Asimismo, la asociación maíz-frijol-calabaza potencia la relación agua-suelo-planta-ambiente, ya que el frijol fija nitrógeno atmosférico que el maíz aprovecha; en tanto que la calabaza con su amplio follaje y hábito rastrero protege al suelo de la 
erosión, promueve la filtración de agua e impide el crecimiento de malezas y la evaporación del agua (Rojas, 1990).

La rotación de cultivos disminuye los problemas de malezas, plagas y enfermedades; aumenta los niveles de nitrógeno disponible en el suelo, reduce la necesidad de fertilizantes sintéticos, y junto con las prácticas de labranza conservadoras del suelo, reduce la erosión edáfica (Altieri y Nicholls, 2000). La conservación de suelos evita la pérdida de nutrientes fundamentales para mejorar capacidades productivas de los suelos agrícolas. Finalmente, el estiércol es un indicador clave de la calidad del suelo ya que provee nutrientes a la planta, mejora la estructura y textura del suelo, aumenta la aireación, penetración y retención de agua, estimula el desarrollo de microorganismos benéficos para la planta y es esencial para capturar carbono (Robert, 2002).

\subsection{La pluriactividad y la sinergia maiz-ganadería}

El manejo del maíz y la sobrevivencia de los productores no se pueden entender sin la sinergia que se establece entre maíz y ganadería, porque el agricultor potencia el empleo de sus recursos ya que le permite:

1. Disponer de energía animal para efectuar distintas prácticas de cultivo. En la entidad 48\% de los maiceros poseen yunta, pero su uso es más común ya que 52, 79 y $80 \%$ la emplean para surcar, dar la primera y segunda labor, de forma respectiva.

2. Contar con un medio de transporte para el acarreo de distintos bienes recolectados de la naturaleza. En el estado, esta actividad es una estrategia de reproducción social esencial, ya que 18, 58, $56,11,68$ y $50 \%$ de los productores extraen de la naturaleza material de construcción (arena, grava, piedra y madera), leña, frutas, animales, plantas comestibles y medicinales, expuestos en el mismo orden.

3. Diversificar sus fuentes de ingresos y alimentación.

4. Reciclar la biomasa y residuos de alimentos que genera la familia del productor.

5. Dotar de estiércol que el productor aplica a su parcela para mejorar las propiedades edáficas de los suelos. En la entidad, 1,251 productores aplicaron abono orgánico y su rendimiento promedio $\left(1,905 \mathrm{~kg} \mathrm{ha}^{1}\right)$ está por arriba del promedio estatal $\left(1,841 \mathrm{~kg} \mathrm{ha}^{1}\right)$.

La estructura ganadera de los tipos de productores se presenta en el cuadro 6 , donde se aprecia que los $\mathrm{P}$, respecto de los POA, poseen más cantidad de ganado mayor, sobre todo vacuno. 


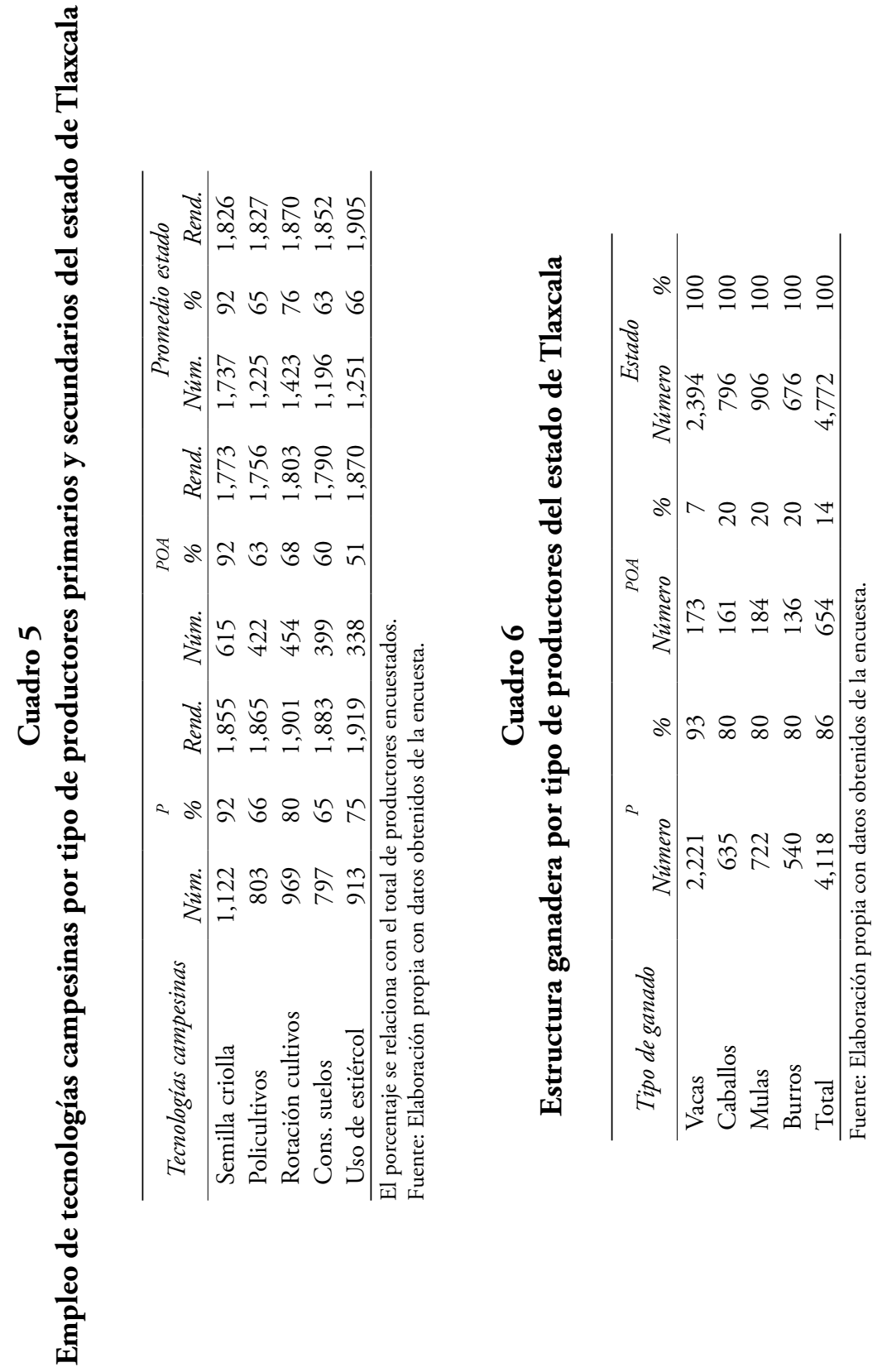




\subsection{Pluriactividad y productividad}

La pérdida de especialización ha ocasionado un manejo deficiente del maíz y baja productividad, que se evidencia en los menores rendimientos promedio que tienen los maiceros del estado, comparados con la media nacional: 1,838 y 2,362 $\mathrm{kg} \mathrm{ha}^{-1}$, de forma respectiva (Sagarpa, 2004). Los mismos datos de la Sagarpa señalan, si se calcula el índice de no siniestro, que los factores edafoclimáticos fueron más favorables para la siembra de maíz en la entidad (0.949) que en el país (0.895). Esto significa que por cada 100 hectáreas sembradas en el estado se siniestraron 5.1, en tanto que esta cifra para el país fue de 11.5. Por tanto, se puede deducir que los bajos rendimientos de los maiceros tlaxcaltecas se deben a la forma en cómo éstos efectúan el manejo del maíz, influenciado notablemente por la pluriactividad que desarrollan.

Es necesario subrayar que el rasgo menores rendimientos por hectárea promedio y condiciones edafoclimáticas más favorables para siembra de maíz, la poseen solamente tres entidades federativas del país: Chiapas, Guerrero y Tlaxcala. Es factible que el comportamiento de los dos primeros se explique por los bajos ingresos que reciben los productores, ya que a partir de 1990 estos estados ocupan los dos primeros lugares en marginación social (Conapo, 2004). Como se sabe, el manejo del maíz está intrínsecamente asociado con la disponibilidad que tienen los productores a los factores de la producción que, a su vez, depende de la capacidad de compra del productor. Pero éste no es el caso de Tlaxcala, catalogada por la misma fuente como de media marginación.

\section{Conclusiones}

La aplicación de las políticas neoliberales en el campo mexicano, y concretamente con productores de maíz, han fomentado la pluriactividad y discontinuidad técnica. En esta investigación se encontró que más de tres cuartas partes de los productores de maíz del estado de Tlaxcala son pluriactivos; que casi dos terceras partes de los maiceros realizan actividades relacionadas con el sector primario, siendo más común este fenómeno entre los maiceros de los DDR 163 y 165; que la pluriactividad ha permitido mayores ingresos de los maiceros, pero al mismo tiempo está afectando la especialización en el manejo adecuado del maíz, así como la productividad de los maiceros, debido a que su discontinuidad técnica se ha ampliado y que la pluriactividad está originando el manejo inadecuado del maíz y el uso incorrecto de agroquímicos, la ruptura de la transmisión de conocimientos empíricos y tecnologías campesinas, así como el deterioro de la sinergia agricultura-ganadería. 


\section{Agradecimientos}

Los autores agradecemos al Sistema de Investigación Zaragoza-Conacyt y al señor Melquíades Pérez González, diputado de la LXVII Legislatura del estado de Tlaxcala, por el financiamiento otorgado para efectuar esta investigación.

\section{Bibliografía}

Altieri, Miguel (1991), “¿Por qué estudiar la agricultura tradicional?”, Agroecología y Desarrollo, 1 (1), Centro Latinoamericano de Desarrollo Sustentable, Santiago de Chile, pp. 16-24.

Altieri, Miguel y Clara Inés Nicholls (2000), Agroecología: teoría y práctica para una agricultura sustentable, Programa de Naciones Unidas sobre Medio Ambiente, México, pp. 13-44.

Anseeuw, Ward y Catherine Laurent (2007), "Occupational paths towards commercial agriculture: The key roles of farm", Journal of Arid Environments, 70 (4), Elsevier Science, Londres, pp. 659-671.

Bartra, Armando (2006), El capital en su laberinto. De la renta de la tierra a la renta de la vida, UACM-ITACA-CEDRSSA, México.

Borlaug, Norman y Christopher Dowswell (2005), "La inacabada Revolución Verde. El futuro rol de la ciencia y la tecnología en la alimentación del mundo en desarrollo", Agbioworld, Alabama, $<$ http://www.agbioworld.org/biotech-info/articles/spanish/desarrollo.html>, 12 de mayo de 2009.

Coplade (Comité de Planeación para el Desarrollo del Estado de Tlaxcala) (2004), Indicadores socioeconómicos de Tlaxcala, Gobierno del Estado de Tlaxcala, México.

Conapo (Consejo Nacional de Población) (2004), Índice absoluto de marginación 1990-2000, Grupo SM Impreso, México.

Damián-Huato, Miguel Ángel, Benito Ramírez Valverde, Abel Gil Muñoz, N. Gutiérrez, A. Aragón, R. Mendoza, Juan Alberto Paredes Sánchez, T. Damián y A. Almazán (2004), Apropiación de tecnología. Características técnicas y sociales de los productores de maiz de Tlaxcala, Benemérita Universidad Autónoma de Puebla, Conse- 
jo Nacional de Ciencia y Tecnología-Sistema de Investigación Zaragoza y H. Congreso del Estado de Tlaxcala, Puebla, México.

Deininger, Klaus y Pedro Olinto (2004), "Empleo rural no agrícola y diversificación del ingreso en Colombia", en NU-CEPAL, Empleo e ingresos rurales no agrícolas en América Latina, CEPAL, Santiago de Chile, pp. 91-106.

Galindo, Arturo (1994), "La agricultura de tiempo parcial en los países industrializados: el caso de Italia", Comercio Exterior, 4 (44) 4, Banco Nacional de Comercio Exterior, México, pp. 313-320.

Gómez-Aguilar, José Roberto (1977), "Introducción al muestreo", tesis de maestría, Colegio de Postgraduados, México.

Grammont, Hubert de (2009), "La nueva estructura ocupacional en los hogares rurales mexicanos", en Hubert de Grammont y Luciano Martínez Valle (coords.), La pluriactividad en el campo mexicano, FLACSO, Quito, pp. 273-303.

Gras, Carla (2004), "Pluriactividad en el campo argentino: el caso de los productores del sur santafecino", Cuadernos de Desarrollo Rural, 51, Pontificia Universidad Javeriana, Bogotá, pp. 91-114.

INEGI-GET (Instituto Nacional de Estadística, Geografía e InformáticaGobierno del Estado de Tlaxcala) (1999), Anuario estadístico de Tlaxcala, Inegi, México.

INEGI-GET (Instituto Nacional de Estadística, Geografía e InformáticaGobierno del Estado de Tlaxcala) (2000), Anuario estadístico de Tlaxcala, INEGi, México.

INEGI-GET (Instituto Nacional de Estadística, Geografía e InformáticaGobierno del Estado de Tlaxcala) (2001), Anuario estadístico de Tlaxcala, INEgi, México.

INEGI (Instituto Nacional de Estadística, Geografía e Informática) (2001), XII Censo de Población y Vivienda del estado de Tlaxcala, INEGI, México. 
INEGI-GET (Instituto Nacional de Estadística, Geografía e InformáticaGobierno del Estado de Tlaxcala) (2002), Anuario estadístico de Tlaxcala, INEGi, México.

INEGI-GET (Instituto Nacional de Estadística, Geografía e InformáticaGobierno del Estado de Tlaxcala) (2003), Anuario estadístico de Tlaxcala, INEGI, México.

INEGI-GET (Instituto Nacional de Estadística, Geografía e InformáticaGobierno del Estado de Tlaxcala) (2007), Anuario estadístico de Tlaxcala, INEGI, México.

INIFAP (Instituto Nacional de Investigaciones Forestales, Agrícolas y Pecuarias) (1999), Programa Rector de Desarrollo Agropecuario 19992005, Base de Datos de Tecnología Agropecuaria del estado de Tlaxcala, INIFAP, México.

Janvry, Alain de y Elisabeth Sadoulet (2004), "Estrategias de ingresos de los hogares rurales de México: el papel de las actividades desarrolladas fuera del predio agrícola", en NU-CEPAL, Empleo e ingresos rurales no agrícolas en América Latina, CEPAL, Santiago de Chile, pp. 107-128.

Martínez, Griselda y Rafael Montesinos (1994), "La innovación organizacional del trabajo", Gestión y Estrategia, 7, Universidad Autónoma Metropolitana-Azcapotzalco, México, <http://www.azc.uam. $\mathrm{mx} /$ publicaciones/gestion/num7/present.htm>, pp. 136-142.

Nadal, Alejandro y Timothy A. Wise (2004). “The Environmental Costs of Agricultural Trade Liberalization of Mexico-U.S. Maize Trade Under nAfTA", Discussion Paper, No. 4, Working Group on Development and Environment in the Americas, <http://ip.cals. cornell.edu/courses/iard6010/mexico/NadalWise July04.pdf>, Brasilia, pp. 24-26.

Ojeda-Trejo, Enrique y Donaciano Ojeda Trejo (1996), "Suelos cultivados de la República Mexicana, contenido medio de nutrimentos minerales aprovechables", México, citado en INEGI-Semarnap, Estadisticas del medio ambiente, Informe de la situación general en materia de equilibrio ecológico y la protección al ambiente, Universidad Autónoma Chapingo, México, p. 80. 
Pengue, Walter (2005), Agricultura industrial y transnacionalización en América Latina. ¿La transgénesis de un continente?, Programa de las Naciones Unidas para el Medio Ambiente-Universidad de Buenos Aires, Buenos Aires.

Roble Pensado-Leglise, Mario del (2005), "La importancia del análisis del gasto público agropecuario y rural en Latinoamérica y México, 1985-2001", Rumbo Rural, año 1/2, Centro de Estudios para el Desarrollo Rural Sustentable y la Soberanía Alimentaria-H. Cámara de Diputados, México, pp. 68-79.

Ponce, Raúl (1998), Zonificación ecológica-económica: una propuesta metodológica para la Amazonia, Tratado de Cooperación AmazónicaSecretaría pro tempore Caracas.

Robert, Michel (2002), Captura de carbono en los suelos para un mejor manejo de la tierra. Informes sobre recursos mundiales de suelos, Organización de las Naciones Unidas para la Agricultura y la Alimentación, Roma.

Rogers, Everett y Lynne Svenning (1979), La modernización entre los campesinos, Fondo de Cultura Económica, México.

Rojas-Rabiela, Teresa (1990), "La agricultura en la época prehispánica”, en Teresa Rojas Rabiela (coord.), La agricultura en tierras mexicanas desde sus origenes hasta nuestros días, Grijalbo-Consejo Nacional para la Cultura y las Artes, México, pp. 15-138.

Rosset, Peter (1997), "La crisis de la agricultura convencional, la sustitución de insumos y el enfoque agroecológico", Agroecología y Desarrollo, 11/12, Centro Latinoamericano de Desarrollo Sustentable, Santiago de Chile.

Rubio, Blanca (2001), Explotados y excluidos. Los campesinos latinoamericanos en la fase agroexportadora neoliberal, Plaza y Valdés, México.

Sacco Dos Anjos, Flavio y Nadia Velleda Caldas (2007), "Pluriactividad y agricultura familiar en Brasil: el caso de Río Grande do Sul”, Revista de la CEPAL, 93, CEPAL, Santiago de Chile, pp. 157-173. 
Sagarpa (Secretaría de Agricultura, Ganadería, Desarrollo Rural, Pesca y Alimentación) (2004), Situación actual y perspectivas del maíz en México: 1990-2003, Sagarpa, México.

SIAP (Servicio de Información y Estadística Agroalimentaria y Pesquera) (2007), Series históricas de superficie sembrada y cosechada, Sagarpa, México.

Semarnat (Secretaría de Medio Ambiente y Recursos Naturales) (2002), Inventario nacional de suelos, Semarnat, México.

Smith, Adam (1982), Investigación sobre la naturaleza y causas de la riqueza de las naciones, Fondo de Cultura Económica, México.

Stern, Nicholas (2007), "The Economics of Climate Change", The Stern Review, Cambridge University Press, Londres, p. 197.

Turrent-Fernández, Antonio, René Camacho Castro, Néstor Francisco Nicolás, Sergio Uribe Gómez, José Isabel Cortés Flores y Ricardo Mendoza Robles (1999), "Posibilidades técnicas de lograr la soberanía alimentaria de origen vegetal en México", Terra Latinoamericana, 17 (1), Sociedad Mexicana de la Ciencia del Suelo, México, pp. 59-76.

Vernimmen, Tom, Marie Bourgeois, Guido van Huylenbroeck, Hennk Meert y Etienne van Hecke (2002), "Diversification as a Survival Strategy for Marginal Farms An Exploratory Research", en $X^{\text {th }}$ EAAE Congress Exploring Diversity in the European Agri-Food System, Zaragoza, Espańa, pp. 9-10.

Recibido: 15 de enero de 2010. Reenviado: 22 de julio de 2010. Aceptado: 25 de noviembre de 2010.

Miguel Ángel Damián-Huato. Es doctor en ciencias en estrategias para el desarrollo agrícola regional por el Colegio de Postgraduados, Campus Puebla; maestro en ciencias en desarrollo rural por la Universidad Autónoma Metropolitana, Xochimilco; ingeniero agrónomo por la Universidad Autónoma de Guerrero. Actualmente es investigador nivel I en el Sistema Nacional de Investigadores (SNI). Sus líneas actuales de investigación son: 
evaluación de la transferencia de tecnología agrícola y manejo sostenible de agroecosistemas, en el Departamento de Agroecología y Ambiente del Instituto de Ciencias de la Benemérita Universidad Autónoma de Puebla (BUAP). Entre sus publicaciones destacan: en coautoría, "Tecnología agrícola y territorio: el caso de los productores de maíz de Tlaxcala, México", Boletin Investigaciones Geográficas, 63, unAm, México, pp. 36-55 (2007); "Hombres y mujeres en la producción de maíz: un estudio comparativo en Tlaxcala”, Región y Sociedad, xx (42), Colegio de Sonora, Hermosillo, pp. 63-94 (2008); en coautoría, "Estrategias de reproducción social de los productores de maíz de Tlaxcala", Estudios Sociales, XviI (34), CIAD, Hermosillo, pp. 111-146 (2009).

Benito Ramírez-Valverde. Es Ph. D. y maestro en estudios latinoamericanos por la Tulane University, Nueva Orleans; también cuenta con una maestría en ciencias en estadística por el Colegio de Postgraduados; es ingeniero agrónomo especialista en fitotecnia, Universidad Autónoma Chapingo. Recientemente fue nombrado investigador nivel iI en el Sistema Nacional de Investigadores (SNI). Sus líneas actuales de investigación son: desarrollo rural, pobreza rural y evaluación de programas de desarrollo, en el Colegio de Postgraduados, Campus Puebla. Entre sus últimas publicaciones destacan: en coautoría, "Tecnología agrícola y territorio: el caso de los productores de maíz de Tlaxcala, México", Boletín Investigaciones Geográficas, 63, unAm, México, pp. 36-55 (2007); "Escuelas de campo y disponibilidad alimentaria en una región indígena de México", Estudios Sociales, XvI (32), CIAD, Hermosillo, pp. 207-226 (2008); en coautoría, "Estrategias de reproducción social de los productores de maíz de Tlaxcala", Estudios Sociales, XviI (34), CIAD, Hermosillo, pp. 111-146 (2009); "Impacto del conocimiento tecnológico sobre la adopción de tecnología agrícola en campesinos indígenas en México", Interciencia, xxxiv (8), Asociación Interciencia, Caracas, pp. 551-556 (2009).

Agustín Aragón-García. Es doctor en ciencias ambientales por el Instituto de Ciencias de la Benemérita Universidad Autónoma de Puebla; maestro en ciencias en biología animal por la Universidad Nacional Autónoma de México; químico farmacobiólogo por la BUAP. Actualmente es investigador nivel I en el Sistema Nacional de Investigadores (SNI). Sus líneas actuales de investigación son: control de plagas con productos alternativos al químico y hábitos y taxonomía de coleópteros Melolonthidae, en el Departamento de Agroecología y Ambiente del Instituto de Ciencias de la BUAP. Entre sus publicaciones destacan: en coautoría, "New saponins from Sechiummexicanum", Magnetic Resonance in Chemistry, 47 (11), Wiley Interscience, Cambridge, Reino Unido, pp. 994-1003, <www.ncbi. 
nlm.nih.gov/pubmed/19637246> (2009); "Identificación de un aislamiento del grupo 16srin, Candidatus Phytoplasma pruni en plantas y semillas de amaranto (Amaranthus hypochondriacus L) en México", Agrociencia, 43 (8), Colegio de Postgraduados, Texcoco, pp. 851-860 (2009); "Descripción de las larvas de tercer instar de Melolonthidae (Coleóptera) asociadas al cultivo de Agave tequilana var. azul y su fluctuación poblacional en Jalisco, México", Neotropical Entomology, 38 (6), Sociedad Entomológica do Brasil, Vacaria, pp. 769-780 (2009).

Jesús Francisco López-Olguín. Es doctor ingeniero agrónomo del Programa de Producción y Protección Vegetal de la Universidad Politécnica de Madrid; es maestro en estadística experimental por el Colegio de Posgraduados, Campus Montecillo; y es ingeniero agrónomo por el Colegio Superior de Agricultura Tropical, Tabasco. Actualmente es investigador nivel I en el Sistema Nacional de Investigadores (SNI). Su línea actual de investigación es manejo agroecológico de sistemas en el cuerpo académico de Ciencias Ambientales y Agricultura y en el Departamento de Agroecología y Ambiente del Instituto de Ciencias de la BUAP. Entre sus publicaciones destacan: en coautoría, "Antifeedant activity and effects of terpenoids on detoxication enzymes of the beet armyworm, Spodoptera exigua (Hübner)", Spanish J. Agric. Res. 6 (special issue), INIA, Madrid, pp. 177-184 (2008); en coautoría, "Estrategias de reproducción social de los productores de maíz de Tlaxcala", Estudios Sociales, XVII (34), CIAD, Hermosillo, pp. 111-146 (2009); "A review of recent patents on macroorganisms as biological control agents", Recent Patents on Biotechnology, 4 (1), Bentham Science, Sharjah, Emiratos Árabes Unidos, pp. 48-64 (2010). 